\title{
El AAI en Uruguay Su aplicación y valoración conceptual por stakeholders
}

Resumen:

El propósito de este documento es presentar los principales resultados de la investigación realizada con el objetivo de replicar el AAI (Active Ageing Index o Índice de Envejecimiento Activo) en Uruguay y evaluar la valoración que de esta herramienta se realiza desde los principales grupos de interés (stakeholders) vinculados a la temática de la vejez y el envejecimiento: Académicos con trayectoria en la temática, Decisores de Políticas Públicas vinculadas a la temática y las Organizaciones de la Sociedad Civil.

\section{Summary:}

The purpose of this document is to in-

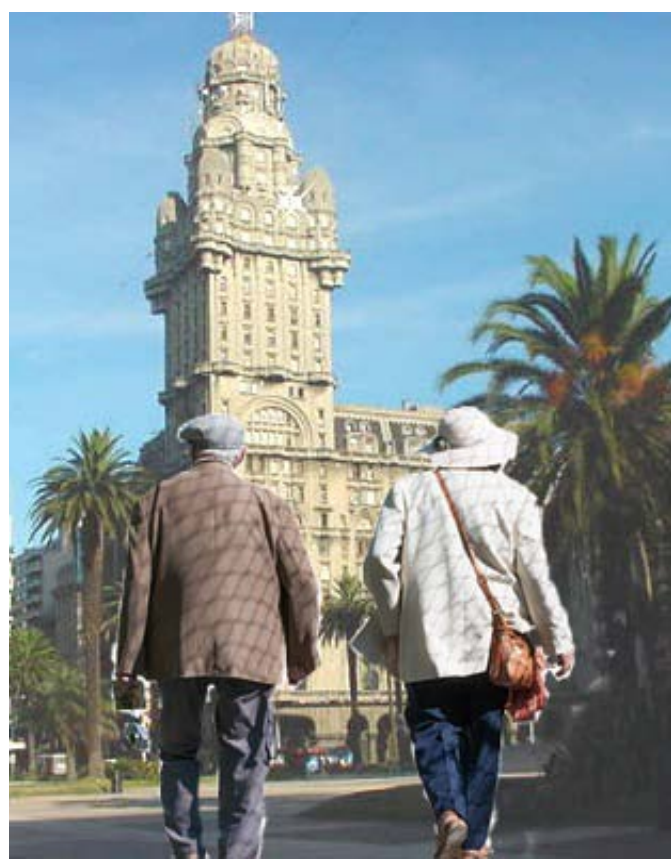
troduce the main results of a research which objective was to replicate the AAI in Uruguay and to evaluate the stakeholders' assessment of it as an appropriate public policy tool. The stakeholders defined for this purpose were: Academics, Policy Makers and Organized Civil Society.
Palabras clave: vejez-envejecimiento activo - políticas públicas
Keywords: old age - active ageing public policies

Maite Ciarniello Miguel: maiteciarniello@gmail.com https://orcid.org/0000-00032913-4806 Magíster en Metodología de la Investigación Científica por la UNLA, Licenciada en Sociología por la UdelaR y Diplomada en Desarrollo Regional y Local por el CLAEH-UCUDAL. Ha desempeñado cargos docentes y de investigación en la UdelaR, en CCSS y Psicología. Actualmente se desempeña en el MTSS de Uruguay. Recibido 09/09/2018, Publicado 27/10/20 


\section{Introducción}

Este artículo presenta los principales resultados de la investigación desarrollada entre 2016 y 2017, cuyo objetivo fue responderse dos preguntas: 1$)$ ¿Es posible replicar el AAI (Active Ageing Index o Índice de Envejecimiento Activo) en contexto diferentes al cual fue creado, en particular en Uruguay? Y 2) ¿Cuál es la valoración que se realiza del AAI desde los stakeholders (grupos de interés) nacionales, como herramienta de política pública, siendo éste su principal objetivo de creación?

Desde nuestra concepción, la primer pregunta nos remite a las condiciones tangibles necesarias para la replicación del AAI en Uruguay: indagar si existen las fuentes de datos adecuadas para obtener la información prevista. Es decir, si esas estadísticas son confiables y están actualizadas, si las preguntas de las encuestas disponibles se adecúan a los objetivos de los indicadores del AAI, etc.

Por otra parte, la segunda pregunta nos remite a las condiciones intangibles, pero necesarias para la replicación del AAI: jes de interés para los stakeholders nacionales el uso de esta herramienta estadística y el tipo de información que ofrece? Nos preguntamo así mismo si existe afinidad teórico-conceptual con el enfoque teórico que sustenta el desarrollo del AAI.

Este artículo presenta los principales resultados a ambas preguntas, además, recoge las contribuciones de los stakeholder relacionadas a la estructura e indicadores que componen el AAI. También muestra el posicionamiento de los representantes de los stakeholders en relación al marco teórico-conceptual que sustenta el enfoque del envejecimiento activo.
Se parte de la concepción de que tanto las condiciones tangibles como las intangibles, son necesarias e inseparables para la replicación de un índice que fue concebido como una herramienta de apoyo al diseño de políticas públicas (en adelante PPPP).

La disponibilidad de información estadística que permita la replicación de los indicadores del AAI en nuestro país es condición necesaria pero no suficiente para sostener en el tiempo el ejercicio de replicación de un índice de estas características. Para lograrlo es necesario además contar con la voluntad y el interés de los stakeholders nacionales.

Por último, y a pesar de que no es el foco de la investigación, se aprovecha la oportunidad para presentar la posición de Uruguay en relación a los rankings disponibles: los países de la Unión Europea y algunos países Iberoamericanos que también han explorado la posibilidad de replicar el AAI de manera local.

\section{Orígenes del AAI}

El AAI, o Índice de Envejecimiento Activo como es su traducción al español, nace en Europa en 2012, en el marco de la celebración del Año Europeo del Envejecimiento Activo y la Solidaridad Intergeneracional y de la Segunda Revisión Quinquenal del Plan de Acción de Madrid.

Es desarrollado por un Grupo de Expertos de la Comisión Económica de Naciones Unidas para Europa (UNECE), con el objetivo de proveer información pertinente, actualizada y comparable entre países, a los Ejecutores de PPPP, con la finalidad de enfrentar los desafíos del envejecimiento poblacional. Identificar las necesidades de la población adulta mayor y potenciar sus capacidades de participación en la sociedad, en el mercado de trabajo y prolongar el mayor tiempo posible una vida independiente y autónoma son algunos de los objetivos para los que esta herramienta aspira a ser de utilidad.

Partiendo del marco conceptual desarrollado por la OMS (2002) el AAI se asienta en la idea de visibilizar los aportes de las personas adultas mayores a la sociedad.

Se trata de un índice compuesto ponderado, integrado por cuatro dominios y 22 indicadores. La definición operacional de envejecimiento activo a partir de la cual se sustenta la herramienta de medición es la siguiente:

Envejecimiento activo refiere a la situación en la que las personas continúa participando en el mercado de trabajo formal al mismo tiempo que se vinculan a otras actividades prodo que se vinculan a otras lavidiespomo la provisión decuidado a miembros de familia y el voluntariado), y viven una vida saludable, independiente y segura a medida que envejecen (Zaidi, 2013)

El uso del AAI se ha expandido en Europa y existen ya algunos ejercicios de replicación en contextos extra-europeos como veremos a lo largo de este artículo, basados en la necesidad de contar con herramientas para manejar realidades poblacionales de naturaleza similar.

En el caso de Uruguay, el país presenta señales de un avanzado grado de envejecimiento poblacional, como ejemplo: según datos de la Encuesta Continua de Hogares de 2017 un 19\% de su población tiene 60 ańos o más.

\section{Abordaje metodológico}

El abordaje metodológico desarrollado consistió en un diseńo mixto, instrumentado en dos etapas correspondientes a cada una de las dos preguntas planteadas inicialmente.

La primera pregunta está basada en la idea de que la estructura metodológica del
AAI es flexible y por lo tanto nos permite trabajar con los llamados indicadores de aproximación, los que respetando la validez de constructo, resultan fuentes apropiadas de información cuando no contamos con los indicadores originalmente propuestos.

Para esta etapa entonces se trabajó en base a un diseńo exploratorio-descriptivo con el objetivo de evaluar la disponibilidad de información estadística en el país en relación a los requerimientos del AAI. Para ello se reunió información de diversas fuentes de datos secundarias, las que presentaron diferentes grados de dificultad, relativa a la unidad de análisis relevada y el periodo de referencia de la encuesta, entre otros.

La segunda pregunta está basada en la concepción de gobernanza y empoderamiento, pilares del enfoque del envejecimiento activo. El AAI, como herramienta de política pública, debe ser evaluado y legitimado en su uso por los llamados stakeholders involucrados: la Academia, los Decisores de PPPP y la Sociedad Civil Organizada.

Con el objetivo de abordar esta dimensión del análisis en esta segunda etapa se implementó un diseño exploratorio-interpretativo en base a una estrategia cualitativa. Es as que a partir de un muestreo intencional de representantes de estos tres grupos de interés se realizó un sondeo para tener una primera aproximación a sus posiciones, opiniones, afinidad conceptual con el abordaje del envejecimiento activo y valoraciones de la herramienta. Para esto se trabajó con la técnica de la entrevista semi-estructurada.

Varios autores sostienen este enfoque teórico, en particular nos apoyamos en Broncano (2006) y su idea del "descubrimiento de la diferencia" a partir de la cual rescata la importancia de poner en pie de igualdad los saberes burocráticos, académicos y cívicos en torno a una temática específica que involucra a varios grupos de interés. Siguiendo esta lógica se optó en esta parte 
de la investigación por un abordaje cualitativo a partir del cual se definieron los tres grupos de interés definidos: la Academia, los Decisores de Política Pública (PPPP) y la Sociedad Civil Organizada.

Las entrevistas se realizaron con la finalidad de indagar acerca del nivel de conocimiento del AAI como herramienta, pero en particular, su afinidad con el enfoque del envejecimiento activo como marco conceptual. Se parte de la idea de que las valoraciones metodológicas y las conceptuales acerca del AAI son indisociables.

\section{El AAI en Uruguay}

Para cumplir con el objetivo de replicar elAAI en Uruguay, se partió de las siguientes premisas: a) en la mayoría de los casos, los indipremisas: a) en la mayoría de los casos, los indipara el AAI no se encuentran disponibles en Uruguay, b) como alternativa se buscaron fuentes de datos secundarias a partir de las cuales obtener indicadores de aproximación que respeten la validez de constructo de los indicadores originales, c) se observó vigilanci epistemológica atendiendo a la confiabilidad de los procedimientos a través de los cuales se obtuvo la información en cada caso. Teniendo en cuenta que se trabajó siempre con fuentes de datos secundarias, se le prestó particular importancia a d) que las fuentes de datos presentaran cobertura nacional, e) que el último valor disponible fuera cercano en el tiempo yf) se tuvo en consideración la frecuencia de actualización prevista teniendo en cuenta que un índice no sólo nos permite visualizarnos en la actualizad en relación a otros, sino que además es una herramienta longitudinal en la medida que nos da información de nuestra propia evolución a lo largo del tiempo. En base a estos parámetros se indagó, depuró y seleccionó una batería de indicadores que dieran cumplimiento a os requerimientos del AAI.

\section{Revista Perspectivas Metodológicas | Universidad Nacional de Lanús | ISSN 2618-4125}

Lo primero que corresponde decir es que efectivamente fue posible replicar el AAI para Uruguay con datos disponibles hasta 2015 en su totalidad, completando la información para los 22 indicadores que componen el AAI.

Sin embargo, no todos los indicadores fueron replicados con el mismo grado de equivalencia en relación a los originales. Siguiendo la clasificación desarrollada por Rodríguez-Rodríguez, V. (2015), solamente 8 de los 22 indicadores fueron replicados de manera "idéntica" a los originales.

Otro grupo de 5 indicadores fueron replicados con pequeños ajustes relativos mayoritariamente a la franja etaria (en función de la disponibilidad de las fuentes) los que llamamos de grado de equivalencia "cercana”, y por último un grupo de 9 indicadores debieron ser modificados, debido a la falta de fuentes de datos, aunque respetando la validez de constructo del indicador original, es decir: la intención original de lo que se busca medir, siendo éstos los llamados indicadores "alternativos o de aproximación".

Otro resultado importante a tener en cuenta es que $8(36,36 \%)$ de los 22 indicadores debieron ser replicados utilizando fuentes de datos que se desarrollaron por única vez, encuestas de implementación puntual que no tienen prevista una nueva edición, lo que atenta claramente con la sostenibilidad en el tiempo de la replicación del AAI.

La siguiente tabla muestra para cada uno de los 22 indicadores del AAI cuál fue la fuente de datos utilizada en Uruguay, el grado de equivalencia del indicador y la frecuencia de actualización prevista de esta fuente de datos.

\begin{tabular}{|c|c|c|c|}
\hline \multicolumn{4}{|c|}{$\begin{array}{l}\text { Fuentes de datos, grado de equivalencia y freceuncia de actualización de los indicadores utilizados } \\
\text { para Uruguay }\end{array}$} \\
\hline Dominios, Indicadores & AAI-Uruguay & $\begin{array}{l}\text { Grado de } \\
\text { equivalencia } \\
\text { del Indica- } \\
\text { dor* }\end{array}$ & $\begin{array}{l}\text { Frecuencia de } \\
\text { actualización del } \\
\text { Indicador para } \\
\text { Uruguay } \\
\end{array}$ \\
\hline \multicolumn{4}{|c|}{ Dominio 1) Empleo (contribución a través de actividades pagas) } \\
\hline 1.1 Tasa de empleo de personas de 55 a 59 años & ECH 2015 & Idéntico & Contínua \\
\hline 1.2 Tasa de empleo de personas de 60 a 64 años & $\mathrm{ECH} 2015$ & Idéntico & Contínua \\
\hline 1.3 Tasa de empleo de personas de 65 a 69 años & ECH 2015 & Idéntico & Contínua \\
\hline 1.4 Tasa de empleo de personas de 70 a 74 ańos & ECH 2015 & Idéntico & Contínua \\
\hline \multicolumn{4}{|c|}{ Dominio 2) Participación en la sociedad (contribución a través de actividades no pagas) } \\
\hline $\begin{array}{l}2.1 \text { Actividad de voluntariado de personas de } 55 \\
\text { años y más }\end{array}$ & EUT 2013 & Cercano & No prevista \\
\hline $\begin{array}{l}\text { 2.2 Cuidado de hijos y/o nietos por adultos-may- } \\
\text { ores de } 55 \text { ańos y más }\end{array}$ & EUT 2013 & Cercano & No prevista \\
\hline $\begin{array}{l}2.3 \text { Cuidado de adultos-mayores por personas de } \\
55 \text { años y más }\end{array}$ & EUT 2013 & Cercano & No prevista \\
\hline $\begin{array}{l}2.4 \text { Participación política de personas de } 55 \text { ańos } \\
\text { y más }\end{array}$ & LB 2015 & Alternativo & Bi-anual \\
\hline \multicolumn{4}{|l|}{ Dominio 3) Vida independiente saludable y segura } \\
\hline 3.1 Ejercicio físico de personas mayores & $\begin{array}{l}\text { Enc. Detección } \\
\text { de Dependen- } \\
\text { cia NIEVE- } \\
\text { MIDES } 2012\end{array}$ & Alternativo & No prevista \\
\hline 3.2 Acceso a la salud y cuidado dental & ECH 2015 & Alternativo & Contínua \\
\hline 3.3 Forma de vida independiente & ECH 2015 & Idéntico & Contínua \\
\hline 3.4 Renta media relativa & ECH 2015 & Cercano & Contínua \\
\hline 3.5 Sin riesgo de pobreza & ECH 2015 & Cercano & Contínua \\
\hline 3.6 Sin privación material severa & ECH 2015 & Alternativo & Contínua \\
\hline 3.7 Seguridad física & LB 2015 & Alternativo & Bi-anual \\
\hline 3.8 Aprendizaje en el curso de vida & EUTIC 2013 & Alternativo & No prevista \\
\hline \multicolumn{4}{|c|}{ Dominio 4) Capacidad y entorno propicio para el envejecimiento activo } \\
\hline $\begin{array}{l}\text { 4.1 Esperanza de vida de } 50 \text { años, a los } 55 \text { años } \\
\text { vividos }\end{array}$ & INE & Idéntico & Contínua \\
\hline 4.2 Esperanza de vida en buena salud & $\begin{array}{l}\text { Elaborado para } \\
\text { esta investiga- } \\
\text { ción en base a } \\
\text { proyecciones } \\
\text { INE }\end{array}$ & Idéntico & No prevista \\
\hline
\end{tabular}




\begin{tabular}{|c|c|c|c|}
\hline 4.3 Bienestar mental & $\begin{array}{l}\text { Enc. Detec- } \\
\text { ción de De- } \\
\text { pendencia } \\
\text { NIEVE- } \\
\text { MIDES } \\
2012 \\
\end{array}$ & $\begin{array}{l}\text { Alterna- } \\
\text { tivo }\end{array}$ & No prevista \\
\hline $\begin{array}{l}\text { 4.4 Uso de TIC (Tecnologías de la infor- } \\
\text { mación y la Comunicación) }\end{array}$ & LB 2015 & $\begin{array}{l}\text { Alterna- } \\
\text { tivo }\end{array}$ & Bi-anual \\
\hline 4.5 Contacto social & $\begin{array}{l}\text { Enc. Detec- } \\
\text { ción de De- } \\
\text { pendencia } \\
\text { NIEVE- } \\
\text { MIDES } \\
2012\end{array}$ & $\begin{array}{l}\text { Alterna- } \\
\text { tivo }\end{array}$ & No prevista \\
\hline $\begin{array}{l}\text { 4.6 Nivel educativo de las personas } \\
\text { mayores }\end{array}$ & ECH 2015 & Idéntico & Contínua \\
\hline \multicolumn{4}{|c|}{$\begin{array}{l}\text { Fuente: Elaboración propia según modelo Rodríguez-Rodríguez } 2015 . \\
\text { *Grado de equivalencia: } \\
\text { Idéntico: idéntico indicador } \\
\text { Cercano: mismo indicador, diferente franja etaria o referencia temporal } \\
\text { Alternativo: otro indicador, respetando validez de constructo }\end{array}$} \\
\hline
\end{tabular}

Si bien no es el foco de la investigación un análisis de la posición del país en los rankings disponibles, a continuación presentamos dos tablas en las que se inserta Uruguay en el ranking europeo 2015 y en el ranking iberoamericano disponible a 2016 como un resultado emergente dela replicación del índice.
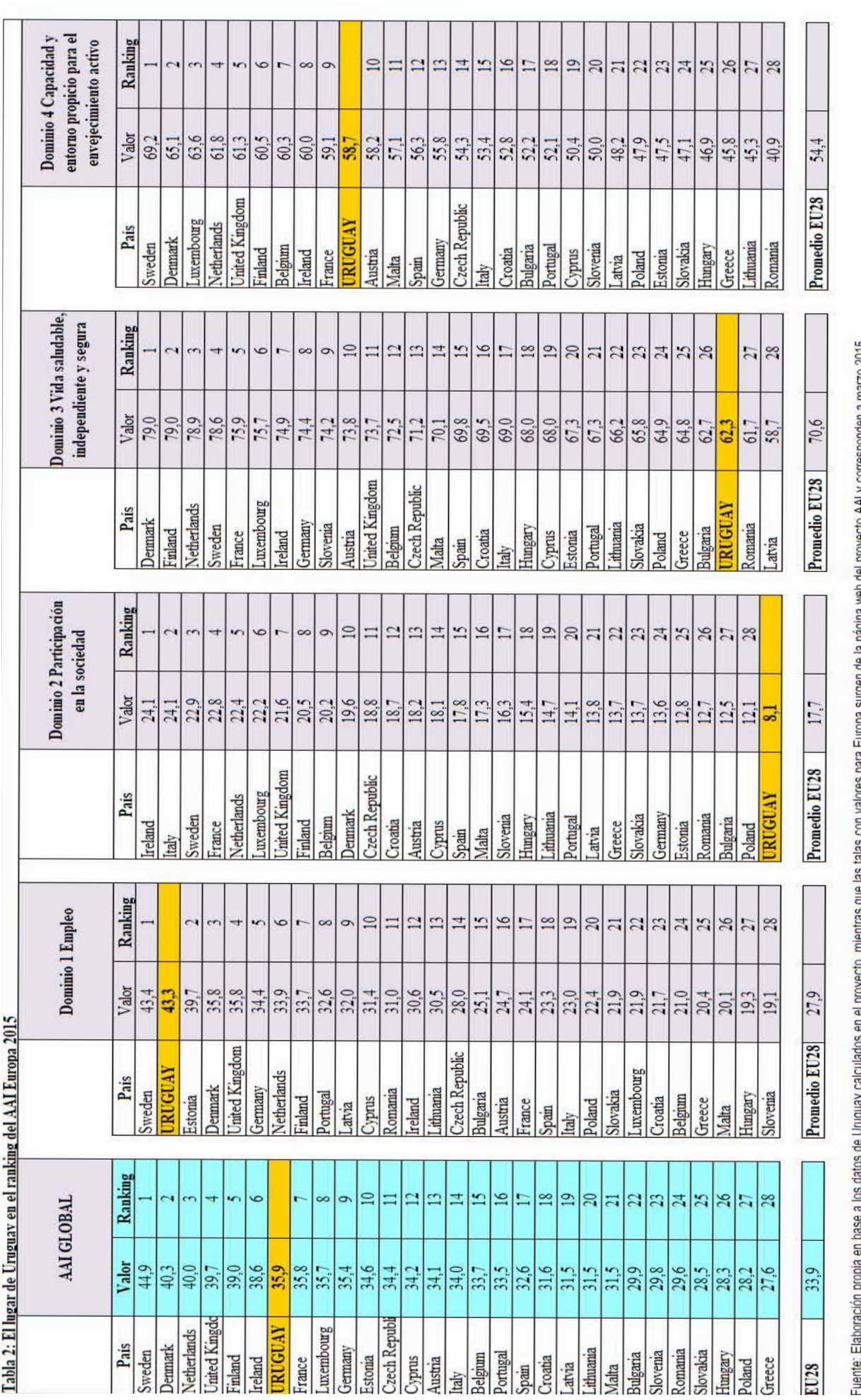

Revista Perspectivas Metodológicas | Universidad Nacional de Lanús | ISSN 2618-4125 
Como se observa, la situación de nuestro país en relación al contexto europeo en términos globales es relativamente buena, ya que Uruguay se posiciona en el primer tercio de países con mejor desempeño. Sin embargo, analizando la situación por dominio, encontramos que en relación al primer dominio, participación en el mercado de trabajo, estamos ubicados en la cima del ranking, pero en los otros dominios el desempeño no es parejo. En particular en el dominio 2, participación en la sociedad, muy por debajo del último país del ranking europeo. Aquí seguramente aspectos metodologicos relativos a la escasez de fuentes de datos para nuestro país y la necesidad de indicadores de aproximación tengan que ver con los resultados.

De cualquier manera, el elemento más interesante es que a pesar de estar al final, incluso muy por debajo, en dos de las cuatro dimensiones o dominios, en el índice globa Uruguay se ubica relativamente bien, y esto se explica por el peso relativo que tiene primer dominio (35\%) en el índice total.

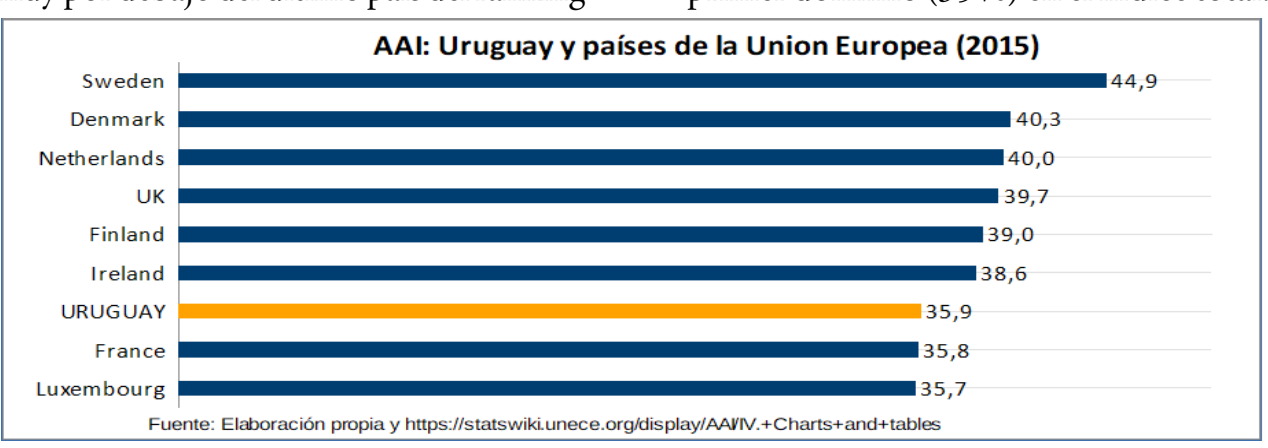

Fuente: Elaboración propia y https://statswikiunece.org/lisplay/AAVN.+Charts and+tables

En relación al contexto iberoamericano, tomando como referencia la publicación realizada por miembros del equipo de la RIIIES en el VII Congreso de ALAP 2016 (Rojo Pérez, F. et al, 2016) se ha elaborado la siguiente tabla.

Tabla 3: Uruguay con paises del contexto Iberoamericano, según datos presentados a VII ALAP 2016

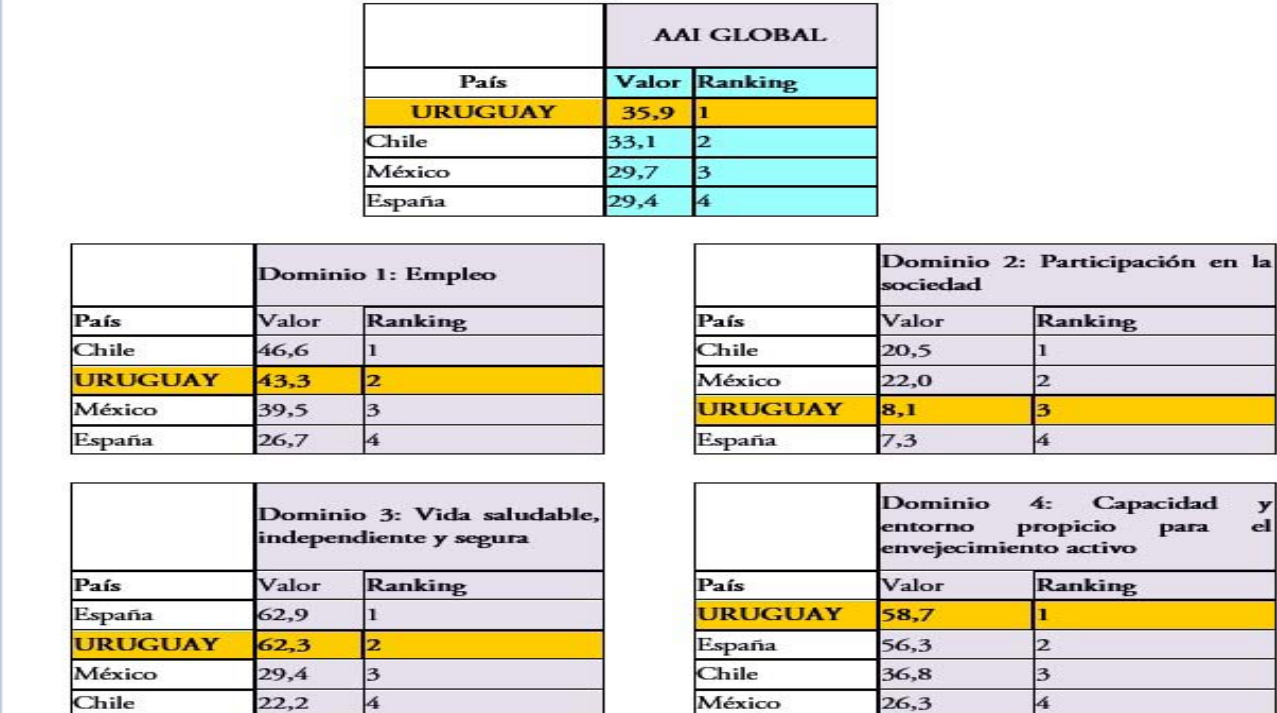

Fuente: Elaboración propia en base a los datos de Uruguay calculados en el proyecto, y los datos para Chile, México y España presentados al VII Congreso ALAP, Foz de Iguazú 2016 (Rojo-Pérez, F. et al 2016).

Revista Perspectivas Metodológicas | Universidad Nacional de Lanús | ISSN 2618-4125
Como se puede ver, Uruguay se encuentra muy bien posicionado en el contexto iberoamericano según lo muestran los datos: en el tope del ranking global y en el medio de los rankings por dominio.

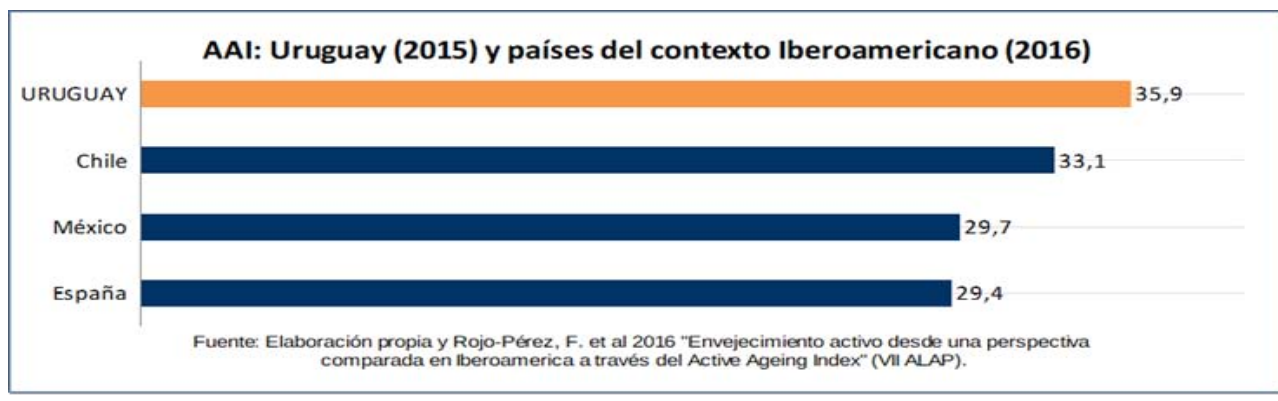

A continuación se presenta una selección de indicadores respecto a los cuales mencionamos algunas consideraciones metodológicas que surgen del ejercicio de replicación realizado:

\section{Indicador 2.4 Participación política}

Identificamos en el indicador original un problema de operacionalización que atenta contra su validez de constructo. El indicador está definido como "participación política" en sentido amplio, y tiene un primer nivel de operacionalización que especifica la participación en sindicatos, partidos políticoso acciones de grupos políticos. En el segundo nivel de operacionalización establece como objetivo relevar actividades de participación que permita a los participantes "influir en las decisiones que se realizan en dichas organizaciones", algo que no está previsto en la definición inicial. Finalmente, en el tercer nivel de operacionalización, en el que se selecciona la pregunta concreta con la que se va a relevar el dato, puede observarse que las actividades de participación que se relevan nada nos dicen de la capacidad de influir del participante en las decisiones de la organización. El ejemplo más claro es la participación en una acción de un grupo político, como podría ser una manifestación o marcha. Parece bastante evidente que

participar en una marcha nada nos dice de la calidad de esa participación en términos de la capacidad de influir del individuo en la toma de decisiones de la organización.

Asimismo, un estudio realizado para Uruguay acerca de la participación de las personas adultas mayores evidencia que la participación en organizaciones se da mayoritariamente en actividades puntuales, siendo una exclusiva minoría, con un sesgo de género importante, la que participa de las instancias de toma de decisiones de dichas organizaciones (Berriel, F. \& Ciarniello M., 2013).

En síntesis, identificamos que en el segundo nivel de operacionalización se in troduce un elemento innovador respecto a la definición original, que luego no es sostenido en el siguiente nivel (y último), y por lo tanto en definitiva no es relevado.

Por último es importante menciona que existen otras formas de participación política que no son a través de los partidos políticos; en particular en el caso de Uruguay existe la REDAM (Red Nacional de Adultos Mayores). La REDAM es una Red nacional de delegados departamentales que tiene participación, establecida por Ley, en el Consejo Consultivo del Instituto $\mathrm{Na}$ cional del Adulto Mayor, del Ministerio 
de Desarrollo Social. Sin embargo, si bien existen estudios de aproximación a esta realidad, no tenemos datos cuantitativo de la participación de las personas mayores en estos ámbitos.

\section{Indicador 3.3 Forma de vida independiente}

Ya en el manual del AAI se aclara que vivir con otras personas no necesariamente implica una pérdida de independencia, e incluso menciona que los arreglos familiares multigeneracionales pueden ser vistos como una forma de vida independiente (Zaidi, A. et al. 2013:52).

Se puede agregar que la independencia en la toma de decisiones sobre la propia vida no está determinada por la conformación del hogar. Que existen numerosos arreglos de cohabitacionalidad que no sólo son el resultado de decisiones autónomas sino que además constituyen la alternativa para mantener la autonomía. Incluso podríamos ir más allá y decir que las personas que viven en instituciones como residenciales, resultado de la propia decisión y financiados con sus propios ingresos, son personas que tienen y mantienen un alto grado de autonomía sobre la propia vida, la que no se pierde por vivir en una residencia colectiva.

Entonces, cabe preguntarse aquí sobre la validez de constructo de este indicador.
En particular si tenemos presente que a veces "vivir solos" no nos hace dueńos de nuestras decisiones, por ejemplo cuando dicha "autonomía" se sustenta en los ingresos económicos de otros miembros de la familia.

Parece que la autonomía sobre las decisiones de la propia vida es un tema que demanda por su naturaleza un abordaje cualitativo, $y$ es difícil obtener datos pertinentes con las encuestas de las que disponemos. Si bien es cierto que estas alternativas son resultado de procesos más modernos e insipientes mientras que tradicionalmente la vida independiente y autónoma se asocia a "vivir solos”, cada vez son más abundantes los estudios que indagan acerca de esta nueva realidad, aunque falta traducir ese conocimiento en información cuantitativa a los efectos de nuestro índice.

Debería pensarse en el tema con un enfoque más complejo y multidimensional que colabore en el desarrollo de encuestas específicas.

\section{Evaluación del AAI como} herramienta de política pública

La segunda pregunta que abordamos atiende a las condiciones de posibilidad pertinencia de la replicación de AAI en Uruguay, dadas por la valoración que del mismo como herramienta de política pública, se realiza desde los stakeholders.

En otras palabras, una vez que sabemos que es posible replicar el AAI en Uruguay en relación a la disponibilidad de información estadística, nos preguntamos si están dadas las condiciones para su replicación como un esfuerzo sostenido en el tiempo, entendiendo que esto solo es posible en la medida que la herramienta sea positivamente valorada y sea de interés sostener el esfuerzo de su replicación.

Esta valoración, como ya se fundamentó, debe provenir de los stakeholders, aquello involucrados de una u otra manera en elaboración, implementación o resultados de esta herramienta.

En los siguientes párrafos presentamos los principales resultados observados. Conviene recordar en este punto que esta parte de la investigación consistió en un trabajo exploratorio del cual no es posible arribar a conclusiones, sin perjuicio de lo cual es enriquecedor como un primer paso para pensar futuros abordajes en nuestro país.
Principales resultados de la segunda parte:

En primer lugar corresponde señalar que surge de las entrevistas que el conocimiento previo que existe del AAI es general y superficial, no hay un manejo profundo de la herramienta y a priori tampoco se identifica un interés explícito en la misma, en particular desde la Academia y los Decisores de PPPP.

Por el contrario, se identificaron manifiestos rechazos a la herramienta y en particular al enfoque teórico-conceptual que la sustenta. Sustentamos esta afirmación en el hecho de que surgen muchas críticas "metodológicas" al AAI a partir del dispositivo de presentación del mismo utilizado en las entrevistas, pero no surgen preguntas respecto a la construcción de los indicadores presentados. Lo que, sumado al desconocimiento del AAI, resulta en crítica muchas veces con argumentos equivocados en relación a la herramienta de medición.

De esta manera, se observa una clara predisposición negativa entre algunos entrevistados (de la Academia y los Decisores de PPPP) frente al AAI, independientemente de su estructura, su contenido y su construcción metodológica de fondo.

Surge de un análisis más profundo de las entrevistas que el elemento disruptivo viene asociado a la terminología utilizada. E término "activo" está fuertemente asociado en la literatura especializada con la "teoría de la actividad” (Salvarezza, L., 2002), que refleja una corriente totalmente contraria al enfoque del envejecimiento activo, pero que genera importantes asociaciones negativas.

Conjuntamente con esta asociación negativa surge la identificación de prácticas que dentro del marco del envejecimiento activo han caído en los típicos errores homogeneizantes y prescriptivos respectos a la vejez y los viejos, típicos del modelo propuesto por la "teoría de la actividad".
Entonces, podríamos decir que algunos entrevistados de la Academia y los Decisore de PPPP condenan el enfoque teórico-conceptual del envejecimiento activo debido a que identifican que las prácticas implementadas desde dicho enfoque, en los hechos han terminado desviándose del mismo.

Una situación diferente se observa con los entrevistados de la Sociedad Civil, que en general no presentan un conocimiento previo del AAI, sin embargo tampoco evidencian rechazo hacia la herramienta sino buena predisposición. Al ser interpelados, los entrevistados de este grupo de interés evidencian una importante sintonía teóricoconceptual con el enfoque del envejecimiento activo, sin embargo, también cuestionan fuertemente las prácticas implementadas desde la políticas públicas, señalando un desfasaje entre discurso e implementación real.

Este grupo reivindica, empoderados desde el paradigma del envejecimiento activo aún sin ser plenamente conscientes de ello, autonomía decisional más allá de la dependencia física eincluso del deterioro cognitivo, respeto y fomento a las decisione personales, la participación para la acción con un sentido subjetivamente determinado y la consulta permanente a las personas adultas mayores en todos los temas que las afectan directa o indirectamente.

Cuando los entrevistados de los tres grupos fueron consultados respecto a la posibilidad de replicar el AAI en Uruguay y si sería una buena herramienta de PPPP los entrevistados dieron sus opiniones en concordancia con sus posiciones respecto al concepto.

Aparecen aquí otros elementos que también influyen en esta valoración, y tienen que ver con qué opinión se tiene de las PPPP actuales y de la disponibilidad de información estadística. Todos los entrevistados coinciden en identificar vacíos de información y la necesidad de generar 
fuentes de información actualizadas en temas de envejecimiento.

Un elemento interesante es el que surge a partir del fuerte énfasis, en particular desde los Decisores de PPPP, con el que se reivindica que cualquier esfuerzo de medición que se lleve adelante como país debe estar orientado a partir de la Convención Interamericana sobre la Protección de los Derechos Humanos de las Personas Mayores, aprobada en la OEA en 2015 y ratificada por Uruguay en 2016. Esto se señala como estrategia país en la medida en que Uruguay ha elegido este camino y los esfuerzos deben potencializarse en esa dirección.

Lo que vuelve interesante esta reivindicación es que la misma se utiliza como argumento en contra del AAI y el marco teórico-conceptual que lo sustenta: el envejecimiento activo.

Sin embargo, la Convención Interamericana sobre la Protección de los Derechos Humanos de las Personas Mayores es un documento en el que el enfoque del envejecimiento activo está explícita e implícitamente presente.

Por su parte desde la Academia se habla de discernir entrelo útil y lo necesario, partiendo de la idea de que cualquier generación de información es útil, pero lo difícil es decidir dónde invertir los recursos siempre escasos. Siguiendo esta línea de pensamiento, es muy interesante el señalamiento que se hace de la Convención Interamericana sobrela Protección de los Derechos Humanos de las Personas Mayores, ya que entendemos que la misma y el AAI no son herramientas conceptuales contrapuestas sino complementarias, que parten de concepciones teórico-conceptuales fuertemente afines, como se desprende de un análisis de ambos documentos, y que profundizar en la línea de la Convención es claramente seguir una estrategia que el país viene perfilando a largo plazo como se señala desde los Decisores de PPPP.
En relación a los aportes y críticas al AAI, su estructura e indicadores que lo componen, identificados por los entrevistados, se destacan las siguientes:

1. Se identifica como un elemento negativo que a la interna del índice se combinen indicadores objetivos con indicadores relativos a la percepción subjetiva de los adultos mayores sobre determinada realidad, aspectos muy variables según la cultura y la idiosincrasia nacional, la influencia de los medios de comunicación, entre otros.

En este punto es fuertemente cuestionado el indicador 3.7 "Seguridad física” por su naturaleza subjetiva, existiendo al ternativas objetivas como cantidad de delitos.

2. En relación a la participación en actividades sociales, en la medida en que es identificada como un elemento central del desarrollo y mejora de la calidad de vida de las personas adultas mayores, se advierte que no está debidamente contemplada en el AAI.

Ya sean actividades de tipo recreativo o cultural o de esparcimiento en general, estas actividades son identificadas por los entrevistados como neurálgicas para la vida en sociedad, y con un fin y sentido en sí mismas dado únicamente por los propios involucrados, sin embargo las mismas no encuentran reflejo en las mediciones del AAI y eso debería corregirse. Además se señala que por su relación con la calidad de vida de las personas el indicador que las comprenda debería ubicarse en el Dominio 4.

3. Otro elemento ausente pero ampliamente reivindicado es la necesidad de medi la imagen social dela vejezy el envejecimiento como un indicador del grado en el que una sociedad habilita, o no, el empoderamiento de sus adultos mayores. De esta manera la imagen social de la vejez constituye un indicador de los entornos propicios para el envejecimiento activo, Dominio 4.
El tema es muy amplio y abarca desde aspectos vinculados al binomio dependencial autonomía, como al binomio económico activo/pasivo, entre otros. En el fondo tiene que ver con el sentido de la acción, con sentirse útil, con ser respetado y con visualizar que no sólo basta con generar los espacios sino también desde la política pública hay que preocuparse por los entornos habilitantes, ya que las imágenes predominantes sobre la vejez y el envejecimiento que existen en una sociedad pueden oficiar como entornos propicios u obstáculos específicos para el ejercicio de un envejecimiento activo.

4. También se identifica que a la hora de medir los entornos (Dominio 4) es pertinente incluir los temas de salud como elementos de base para el efectivo ejercicio de derechos. En esta misma línea se incluye el acceso a la justicia y a la administración pública en tiempos breves, acordes a la etapa de la vida en la que se encuentran las personas adultas mayores, elemento que el AAI no contempla y que está en línea con la Convención Interamericana sobre la Protección de los Derechos Humanos de las Personas Mayores de la OEA.

5. Por último, decir que el indicador 4.2 "Esperanza de vida en buena salud" es altamente valorado desde la Academia y los Decisores de PPPP. Se señala la ausencia de este dato a nivel nacional, la necesidad de contar con él, pero con la salvedad de evaluar los costos que pueda implicar y la importancia de que su cálculo sea asumido de manera sistemática por organismos confiables como la Academia o el Instituto Nacional de Estadística.

\section{Reflexiones finales}

A pesar de que el país ha realizado importantes compromisos en materia de política internacional con el enfoque de política del envejecimiento activo, surge de las entrevistas realizadas que actualmente no podemos afirmar que este enfoque sea ampliamente aceptado en nuestro país.

Puntualmente, en relación a la herramienta que nos convoca: el AAI, si bien ha sido posible obtener información para el total de los 22 indicadores que lo componen (con las aclaraciones oportunamente consignadas), a la luz de las entrevistas realizadas no se puede afirmar que exista en términos generales una valoración positiva de la herramienta, aunque tampoco puede afirmarse lo contrario. Ocurre que entre los entrevistados existen posiciones diversas, observándose mayor afinidad con el AAI entre los representantes de las Organizaciones de la Sociedad Civil, que entre los otros dos grupos de interés, sin presentar los entrevistados de los Decisores de PPPP y los entrevistados de la Academia una postura homogénea a su interna.

Para que el AAI pueda convertirse en una herramienta útil para nuestro país consideramos que debería tenerse en cuenta lo siguiente:

1. Actualizar encuestas específicas que fueron realizadas por única vez, pero que son fuente de información muy valiosa en relación no sólo a la replicación de esta herramienta sino a la temática en general,

2. Superar las resistencias identificada entre los Académicos y los Decisores de PPPP, para lo cual es fundamental tener en cuenta las recomendaciones de medición realizadas por ambos, y profundizar en el análisis de los significados simbólicos asociados a la terminología, así como de las implicancias del enfoque del envejecimiento activo como marco de PPPP.

Finalmente, es importante recordar que como surge del manual del AAI, uno de los 
objetivos de este enfoque es: remain in charge of our own lives as long as possible (Zaidi, A. et al, 2013), y para ello el empoderamiento, el sentido de sí mismos y nuestro sentido de la acción es central, por lo que estos elementos, sin ninguna duda, deben estar presentes de alguna manera en una medición que intente dar cuenta del grado de envejecimiento activo de una sociedad.

\section{Bibliografía}

- Alfama, E., Canal, R., \& Cruells, M. (2013). Las políticas de envejecimiento activo en el Estado español (2002-2012) ¿promoviendo la ciudadanía y la participación de las personas mayores? En IV Congreso REPS, Alcalá de Henares.

- Batthyany, K., Berriel, F., Carbajal, M., Genta, N., Paredes, M., Pérez Fernández, R., \& Perrotta, V. (2014). Las personas mayore ante el cuidado. Aportes de Inmayores para la construcción de un sistema Nacional de Cuidados. Montevideo: Sistema Nacional de Cuidados, INMAYORES, MIDES. ISBN : 978-9974-715-31-8

- Berriel, F. (coord.) \& Ciarniello, M. (2013). Relevamiento nacional de organizaciones de adultos mayores con participación en la Red Nacional de Organizaciones de Adultos Mayores (REDAM). (Informe final de investigación). Montevideo, MIDES-NIEVE (Inédito).

- Broncano, F. (2006). Entre ingenieros y ciudadanos. Filosofía de la técnica para días de democracia. Barcelona: Montesinos.

- Calvo, J. J. (2010). Nociones básicas de demografía. Montevideo: CECEA.

-Carbajal, M. (2014). Los significados del cuidado desde la perspectiva de las personas adultas mayores. Estudio cualitativo en la ciudad de Montevideo. (Tesis de Maestría en Psicología Clínica). Facultad de Psicología, Universidad de la República, Montevideo.
- Carbajo Vélez, M.C. (2009). Mitos y estereotipos sobre la vejez. Propuesta de una concepción realista y tolerante. En ENSAYOS Revista de la Facultad de Educación de Albacete, No24, 2009 (p. 87-96). ISSN 2171-9098

- CELADE (2006). Manual sobre indicadores de calidad de vida en la vejez, Documentos de proyectos n. ${ }^{\circ} 113$, Santiago de Chile, CEPAL.

- CELADE-CEPAL (2004). Estrategia Regional de Implementación para América Latina y el Caribe del Plan de Acción Internacional de Madrid sobre Envejecimiento. Santiago de Chile: UNFPA.

- Ciarniello, M. (Junio, 2018). El Índice de Envejecimiento Activo en América Latina. Su aplicabilidad y valoración conceptual desde actores relevantes del Estado, la Academia y la Sociedad Civil. El caso de Uruguay. (Tesis de Maestría en Metodología de la Investigación Científica). Departamento de Humanidades, Universidad Nacional de Lanús, Buenos Aires. (http://repositoriojmr. unla.edu.ar)

- Ciarniello, M. (Octubre, 2013). La Red Nacional de Adultos Mayores (REDNAM): un proceso social de interpelación de lo político impulsado desde el propio Estado. En XXIX Congreso ALAS. Crisis y emergencias sociales en América Latina. Santiago de Chile.

- Consejo Consultivo del Instituto Nacional de las Personas Mayores (2016). Segundo Plan Nacional de Envejecimiento y Vejez 2016-2019. Montevideo: Ministerio de Desarrollo Social, Instituto Nacional de las Personas Mayores. ISBN: 978-9974715-54-7

- Consejo Consultivo del Instituto Nacional del Adulto Mayor - INMAYORES (2012). Plan Nacional de Envejecimiento y Vejez 2013-2015. Montevideo: Ministerio de Desarrollo Social.
- Cumming, E., \& Henry, W. (1961). Growing old. New York: Basic.

- Fanta Garrido, J. (2015). Quality of Life of the Elderly and Applicability of the Active Aging Index to Latin American Countries. Argentina, CONICET.

- Flick, U. (2007). Introducción a la investigación cualitativa (Segunda edición). Madrid: Ed. Morata. ISBN 978-84-7112 480-7

- Glaser, Barney G \& Strauss, A. (1967) The discovery of Grounded Theory strategic for qualitative research. Aldine Publishing Company, New York.

- Havinghurst, R. (1961). Succesful aging. En The Gerontologist 1, pp 230-236.

- Help Age Internacional (2013). Índice Global de Envejecimiento, AgeWatch 2013 Propósito, metodología y resultados. Londres. www.globalagewatch.org

- Hermida, P. \& Stefani, D. (2011) La jubilación como factor de estrés psicosocial. Un análisis de los trabajos científicos de las últimas décadas. En Perspectivas en Psicología Vol. 8 - Noviembre 2011 (pp. 101-107).

- Iacub, R. (2002). La postgerontología: hacia un renovado estudio de la gerontología. En Revista Latinoamericana de Psicogerontología vol.34,pp: 155-157. Fundación Universitaria Konrad Lorenz. Bogotá, Colombia. ISBN 0120-0534

- INE (2014). Estimaciones y proyecciones de la población de Uruguay: metodología y resultados. Disponible en http://www. ine.gub.uy/c/document_library/get file? unid=c4d937f9-49e $4-4989-\mathrm{b} 3 \mathrm{fc}-$ c6130745233b\&groupId $=10181$

- INE. Comisión Económica para América Latina y el Caribe Oficina de Montevideo (1996). ASPECTOS METODOLOGICOS SOBRE MEDICION DE LA LINEADE POBREZA: el caso uruguayo. Documentos y Resumen Ejecutivo del Taller Regional de Expertos sobre Medición de la Línea de Pobreza en Uruguay, 12 al 13 de marzo, 1996. Disponible en: http://www.ine.gub. uy/documents/10181/36026/

Aspectos+Metodol $\%$ C3\% B3gicos+sobre+medici $\% \mathrm{C} 3 \% \mathrm{~B} 3 \mathrm{n}+\mathrm{de}+\mathrm{la}+1 \%$ C3\%ADnea+de+Pobreza.pdf/d812b951 d975-48bd-a9a6-77264da04715

- IMSERSO (2011). Libro Blanco del Envejecimiento Activo. Ed. Ministerio de Sanidad, Política Social e Igualdad Secretaría General de Política Social y Consumo. Instituto de Mayores y Servicios Sociales (IMSERSO). España.

- Ley 18.617 Instituto Nacional del Adulto Mayor. Publicación: 06/11/2009. Registro Nacional de Leyes y Decretos: Tomo: 1, Semestre: 2, Año: 2009, Página: 1296. (2009).

- Manes, R. (2014). Participación educativa universitaria y estado de la salud percibido en personas mayores: estudio cuali-cuantitativo en los cursos y talleres dependientes de la DEP-UNLA [en Línea]. Lanús. Departamento de Humanidades y Artes. Disponible en:

http://www.repositoriojmr.unla.edu.ar/ descarga/Tesis/MAMIC/Manes_R_Participacion_2014.pdf

- Mayoralas-Fernández, G., Rojo-Pérez, F., Prieto-Flores, M., Forjaz, M., Rodríguez-Rodríguez, V., Montes de Oca, V. (...) \& Mayoral-Pulido, O. (2014). Revisión conceptual del envejecimiento activo en el contexto de otras formas de vejez. XIV Congreso Nacional de Población, AGE. Sevilla.

- Naciones Unidas, CEPAL (2012) Carta de San José sobre los derechos de las personas mayores de América Latina y el Caribe. Adoptada en la tercera Conferencia regional intergubernamental sobre envejecimiento en América Latina y el Caribe. San José de Costa Rica, 8 
al 11 de mayo de 2012 Disponible en: http://repositorio.cepal.org/bitstream/ handle/11362/21534/S2012896 es.pdf?sequence $=1$

- Naciones Unidas, CEPAL (2011). Declaración de Brasilia. Segunda Conferencia regional intergubernamental sobre envejecimiento en América Latina y el Caribe: hacia una sociedad para todas las edades y de protección social basada en derechos. Brasilia, 4 al 6 de diciembre de 2007. Disponible en: https://www.cepal.org/publicaciones/ xml/0/32460/LCG2359_e.pdf

- Naciones Unidas (2003). Declaración Política y Plan de Acción Internacional de Madrid sobre el Envejecimiento. Nueva York, Naciones Unidas.

- OEA (2015). Convención Interamericana sobre la protección de los derechos humanos de las personas mayores. Asamblea General, Resolución 2875 (XLV-0/15). Washington D.C.

- OMS (2002). Envejecimiento activo: un marco político. En Revista Especializada en Geriatría y Gerontología. 2002; 37 (S2): 74-105.

- Paredes, M., Montes de Oca, V., Rodríguez, V., Berriel, F., Lladó, M. \& Guidotti, C. (Octubre, 2016). Envejecimiento y derechos: una visión comparativa desde las agendas políticas de tres países. En VII Congreso de la Asociación Latinoamericana de Población (ALAP) y XX Encontro Nacional de Estudos Populacionais. Foz de Iguazú, Brasil.

- Paredes, M. \& Ciarniello, M. (Noviembre 2013). Participación social de las personas adultas mayores en Uruguay, estudio cuantitativo. En II Congreso Latinoamericano de Gerontología Comunitaria, Buenos Aires.

- Paredes, M. (coord.), Pérez, R., González Arias, D. \& Guidotti González, C.
(2013). Encuesta de detección de población adulta mayor dependiente. (Informe final de investigación). Montevideo, MIDES-NIEVE (Inédito).

- Paredes, M., Ciarniello, M. \& Brunet, N. (2010). Indicadores sociodemográficos de envejecimiento y vejez en Uruguay: Una perspectiva comparada en el contexto latinoamericano. Montevideo. UdelaR-UNFPA. Lucida Ediciones. ISBN: 978-9974-0-0668-3.

- Pellegrino, A. et al. (2008). De una transición a otra: la dinámica demográfica del Uruguay en el siglo XX. En El Uruguay del SXX. La sociedad. Montevideo, Departamento de Sociología-FCS-Ediciones de la Banda Oriental.

- Pugliese. L. (2014). El Envejecimiento Activo, aproximaciones conceptuales y ejemplos de programas que lo promueven. (Asesoría en Políticas de Seguridad Social. Asesoría General en Seguridad Social) Montevideo: Banco de Previsión Social.

- Quiñones, M, Supervielle, M \& Acosta, M. (2017). Introducción a la sociología cualitativa: fundamentos epistemológicos y elementos de diseño y análisis. Montevideo: UdelaR. CSEP

- Rodríguez-Rodríguez, V., Rojo-Pérez, F., Fernández-Mayoralas, G., Morillo, R., Forjaz, J. \& Prieto-Flores, M. (2015). Active Ageing Index: Application to spanish geographical scales. International Seminar. Building an evidence base for active ageing policies: Active Ageing Index (AAI) and its potential. Brussels.

- Rojo-Pérez, F., Garay Villegas, S., Rodríguez-Rodríguez, V., Santos Coria, A. N., Fernández-Mayoralas, G., Montes de Oca, V., Herrera, S. \& Fernández, B. (Octubre, 2016). Envejecimiento activo desde una perspectiva comparada en Iberoamérica a través del 'Active Ageing
Index'. En VII Congreso de la Asociación Latinoamericana de Población (ALAP) y XX Encontro Nacional de Estudos Populacionais. Foz de Iguazú, Brasil.

- Salvarezza, L. (2002). Psicogeriatría. Teoría y clínica. Buenos Aires: Ed. Piados.

- Samaja, J. (2012). Epistemología y metodología: elementos para una teoría de la investigación científica. 3a ed. 9a reimp.- Buenos Aires: Eudeba. ISBN 978-950-23-0931-6

- Villareal Martínez, M. (2006). Mecanismos participativos en el diseño, formulación e implementación de leyes políticas y programas sobre envejecimiento. Santiago de Chile. Serie Población y desarrollo, 70. CEPAL. UNFPA, Naciones Unidas.

- Ynoub, R. (2014). Cuestión de método. Aportes para una metodología crítica. Tomo I. México: Cengage Learning. ISBN: 978-987-1954-48-3

- Zaidi, A. (2015). Active Ageing Index: A Legacy of the European Year 2012 for Active Ageing and Solidarity between Generations. European Center for Social Welfare Policy and Research.

- Zaidi, A. (2015). Active Ageing Index 2014: Analytical Report. ONU, Economic Commission for Europe.

- Zaidi, A., Gasior, K., Hofmarcher, M., Lelkes, O., Marin, B., Rodrigues, R. (...) \& Zolyomi, E. (2013). Active Ageing Index 2012 Concept, Methodology and Final Results. Research Memorandum/ Methodology Report. Viena, European Centre Vienna. Disponible en: http://www.euro.centre.org/data/ aai/1253897823_70974.pdf

- Zaidi, A. et al. (Eds.). Building Evidence for Active Ageing Policies. Acti- ve Ageing Index and its Potential. (en prensa).

Links de referencia:

http://www.cardiosalud.org/files/documents/chscv-encuesta-2005-habitos-deportivos.pdf

http://www.envejecimientoysociedad. com/es/home/

Instituto Nacional de Estadística de España. Metodología para el cálculo de esperanzas de vida en salud. Disponible en: http://www.ine.es/daco/ daco42/discapa/meto.pdf 